\title{
Clinical performance of house-dust-mite-specific subcutaneous immunotherapy in a postmarket noninterventional setting
}

\author{
Petra Zieglmayer · Ralph Mösges · Silke Allekotte · Sandra Weissenbaeck • Michael Moser · Cengiz Acikel · \\ Esther Raskopf · Matthias F. Kramer · Katrin Birkholz · Sonja Guethoff
}

Received: 19 August 2020 / Accepted: 12 September 2020 / Published online: 20 October 2020

(C) The Author(s) 2020

Keywords Microcrystalline tyrosine - Safety · Tolerability · Clinical effect · Updosing

Abbreviations

ADR Adverse drug reaction

AR Allergic rhinitis

HDM House dust mite

MCT Microcrystalline tyrosine

NIS Non-interventional study

NS Not significant

SCIT Subcutaneous allergen-specific immunotherapy

SD Standard deviation

SU Standard units

V Visit

\section{To the editors}

Clinical premarket trials evaluate efficacy and safety of a therapeutic compound in highly selected participant groups under closely monitored circumstances. In contrast, noninterventional studies (NIS) on marketed products are often considered inferior, but are nevertheless encouraged by authorities, especially in terms of safety of real-life patients.

Here, we analysed the tolerability and safety during the updosing phase of a house dust mite (HDM) subcutaneous allergen-specific immunotherapy (SCIT) $[1,2]$, marketed in Austria shortly before start of this study.

The open prospective NIS was conducted in Austria and ran in 7 centres from 2017 to 2019. After having given written informed consent, 32 eligible patients including 5 children (Online Resource 1)

\section{S. Weissenbaeck}

Medical Practice Dr. Weissenbaeck, Vienna, Austria

\section{Moser}

Medical Practice Dr. Moser, Linz, Austria

K. Birkholz · S. Guethoff

Bencard Allergie GmbH, Vienna, Austria

M. F. Kramer · K. Birkholz · S. Guethoff Bencard Allergie GmbH, Munich, Germany

M. F. Kramer

Allergy Therapeutics, Worthing, UK

C. Acikel · E. Raskopf

ClinCompetence Cologne GmbH, Cologne, Germany 
Table 1 Tolerability of the treatment as assessed by the investigator and the patient

\begin{tabular}{|c|c|c|c|c|c|c|}
\hline \multirow[t]{2}{*}{ Visit } & \multirow[t]{2}{*}{ Dose [SU] } & \multicolumn{2}{|l|}{ Adverse drug reactions } & \multicolumn{3}{|l|}{ Tolerability } \\
\hline & & Type $(n)$ & Description & Very good & Good & Poor \\
\hline 1 & 150 & - & - & $100.0 \%$ & - & - \\
\hline 2 & 300 & $\begin{array}{l}\text { Mild local reaction (1) } \\
\text { mild to moderate systemic reaction (1) }\end{array}$ & $\begin{array}{l}\text { Swelling } \\
(5-10 \mathrm{~cm}) \\
\text { recurrent airway obstruction }\end{array}$ & $93.8 \%$ & $3.1 \%$ & $3.1 \%$ \\
\hline $2 b^{\mathrm{a}}$ & 600 & - & - & $100.0 \%^{b}$ & - & - \\
\hline 3 & 900 & Mild local reaction (2) & $\begin{array}{l}\text { Swelling } \\
(5-10 \mathrm{~cm})\end{array}$ & $93.1 \%$ & $6.9 \%$ & - \\
\hline $3 b^{\mathrm{a}}$ & 1200 & - & - & - & $100.0 \%^{b}$ & - \\
\hline 4 & 1500 & - & - & $93.1 \%$ & $6.9 \%$ & - \\
\hline 5 & 1500 & - & - & $93.1 \%$ & $6.9 \%$ & - \\
\hline
\end{tabular}

with allergic rhinitis (AR) with/without (+/-) asthma were treated with purified, modified and microcrystalline tyrosine (MCT)-adsorbed allergen extracts of HDM Dermatophagoides pteronyssinus and/or Dermatophagoides farinae. The study comprised 6 visits (V1-V6) including 4 visits during the short updosing phase (Online Resource 2). Patient characteristics (Online Resource 3), diagnosis of AR, symptoms and medication use (Online Resource 4) in the previous year as well as tolerability and medication use and symptoms after initiation of HDM-SCIT were evaluated.

Overall, no fatality nor serious adverse drug reaction (ADR) occurred during the study. No epinephrine was used. Four ADRs were reported in 3 adults (9.3\%). One asthmatic patient with a mild to moderate recurrent airway obstruction classified as grade II systemic reaction discontinued the treatment. Swelling at the injection site $(5-10 \mathrm{~cm}$ in diameter) occurred in 3 patients during the updosing phase: 1 reaction at 300 standard units (SU) and 2 at 900 SU. Tolerability was mainly rated 'very good' as assessed by the investigators and patients (Table 1).

All symptom scores were lower (by up to $56.3 \%$ ) at V6 in comparison to the retrospective evaluation at V1 (Fig. 1). The individual symptoms 'sneezing' and 'nasal obstruction' improved the most (Fig. 1, Online Resource 5). Symptomatic medication use was also substantially reduced (Online Resource 6).

Here, we show that the HDM-SCIT is safe and well tolerated during the updosing phase in the scope of standard medical care. ADRs occurred after $2.6 \%$ of all injections, which is in line with data from a recent meta-analysis on SCIT [3]. However, of the originally planned 100 participants only 32 patients were included in this study due to an unexpectedly low participation rate of the study centres. The main barrier holding off investigators from participating was the administrative work load to fulfil requirements of the Vienna ethics committee which was comparable to clinical trials.
At the same time, the conduct of NIS is encouraged by regulatory authorities and the community, to demonstrate the clinical use of marketed products in unselected real-life patients. In practice, however, the bar is set so high that the efforts required to participate in a NIS is almost similar to those required for a clinical trial. Hence, only physicians who are experienced in clinical trials and are therefore not afraid of the administrative burden of such a study, are willing to participate. Moreover, the general negative image of NIS also prevented us from reaching the targeted number of included patients.

Despite the low inclusion figures, clinical effects on allergic symptoms were already detected that are comparable to a recent study presenting data after 2 years of treatment [4]. The use of symptomatic medication was also considerably reduced.

In conclusion, the modified and MCT-adsorbed HDM-SCIT showed no elevated application risk during updosing in conjunction with favourable clinical effects in the majority of patients. Since NIS can represent a cross-section of real-life patients, it should be considered complementary to clinical trials, and therefore, common acceptance and feasibility of conducting NIS would be desirable.

Funding This study was funded by Bencard Allergie GmbH.

Authors' contributions R. Mösges, S. Allekotte, M. F. Kramer, and S. Guethoff designed the study; S. Allekotte, K. Birkholz, and S. Guethoff performed the study supported by the local investigators; P. Zieglmayer, S. Weissenbaeck, M. Moser contributed to the study as local investigators. R. Mösges, S. Allekotte, K. Birkholz and S. Guethoff analysed the data; P. Zieglmayer, R. Mösges, E. Raskopf, S. Allekotte, K. Birkholz, and S. Guethoff wrote the paper. E. Raskopf prepared the figures. All authors have read, discussed and approved the final version of the manuscript.

Funding Open Access funding enabled and organized by Projekt DEAL. 


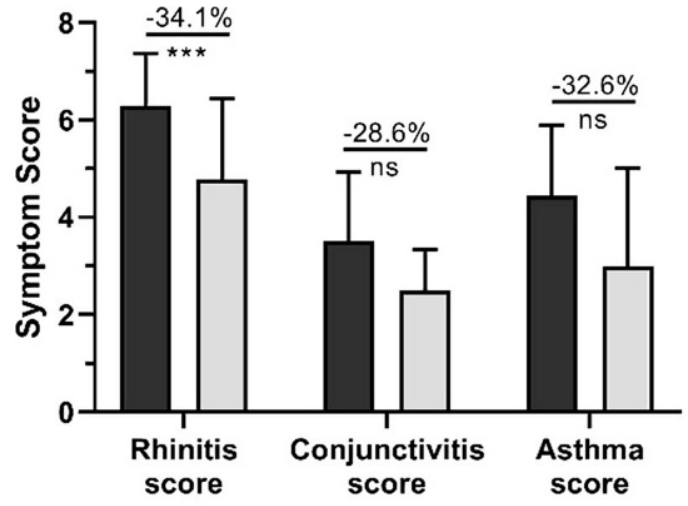

a

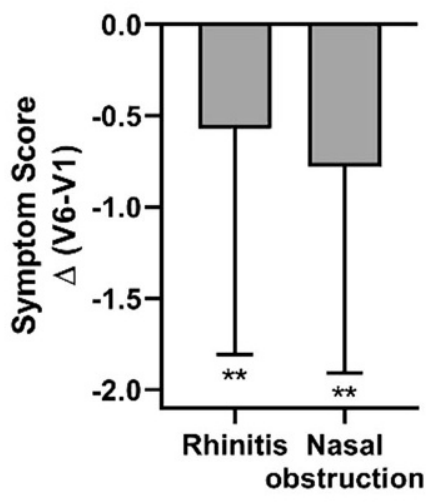

b

Fig. 1 Symptom scores from visit 1 (V1) to visit 6 (V6). The rhinitis, conjunctivitis and asthma scores (1-7 points), combining symptom severity and symptom frequency, were assessed retrospectively at visit $1(N=31, N=10, N=11$, respectively) and after the updosing phase (subcutaneous allergen-specific immunotherapy [SCIT] initiation) at $\mathrm{V} 6(N=18, N=6, N=6$, respectively). The data are presented as mean + standard deviation (SD), ${ }^{\star * *} p=0.002$ (a). The reduction was even more pronounced when comparing patients who had evaluations at V1 and V6 $(N=18, N=5, N=5$, respectively), with $-34.1 \%$ for rhinitis, $-50.0 \%$ for conjunctivitis and $-56.3 \%$ for asthma

\section{Compliance with ethical guidelines}

Conflict of interest R. Mösges and S. Allekotte are employees of CRI-Clinical Research International Ltd. which served as the clinical research organisation for this study. E. Raskopf and C. Acikel were employees of CRI-Clinical Research International Ltd. at the time the study was conducted. R. Mösges reports personal fees from ALK, grants from ASIT biotech, personal fees from Allergopharma, personal fees from Allergy Therapeutics, grants and personal fees from Bencard Allergie $\mathrm{GmbH}$, grants from Leti, grants, personal fees and non-financial support from Lofarma, non-financial support from Roxall, grants and personal fees from Stallergenes, grants from Optima, personal fees from Friulchem, personal fees from Hexal, personal fees from Servier, personal fees from Klosterfrau, non-financial support from Atmos, personal fees from Bayer, non-financial support from Bionorica, personal fees from FAES, personal fees from GSK, personal fees from MSD, personal fees from Johnson\&Johnson, personal fees from Meda, personal fees and non-financial support from Novartis, non-financial support from Otonomy, personal fees from Stada, personal fees from UCB, non-financial support from Ferrero, grants from BitopAG, grants from Hulka, personal fees from Nuvo, grants from Ursapharm, outside the submitted work. S. Allekotte reports grants and personal fees from Lofarma, personal fees from Servier, personal fees from Hexal, personal fees from Friulchem, outside the submitted work. E. Raskopf reports personal fees from Bayer and personal fees from Gesellschaft für Phytotherapie outside the submitted work. P. Zieglmayer received lecture fees by Alk Abello, Allergopharma, Allergy Therapeutics, Novartis, Stallergenes, Thermo Fisher Scientific, and received scientific and educational grants by Allergopharma, Allergy Therapeutics, Biomay, Calistoga, GSK, HAL, MSD, Ono, Oxagen, RespiVert, Stallergenes and VentirX outside the submitted work. S. Weissenbaeck and M. Moser participated as investigators in this study. K. Birkholz, M. F. Kramer and S. Guethoff are employees of Bencard Allergie GmbH, Vienna, Austria and/or Munich,

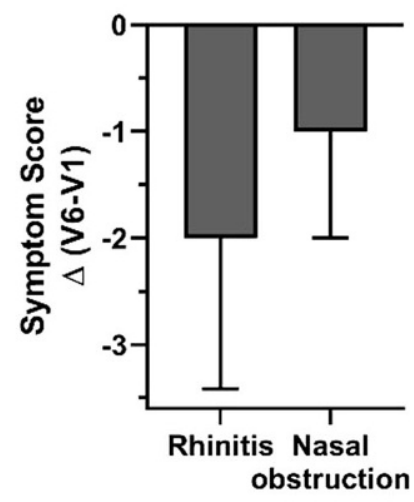

c

symptoms. The severity of the pronounced nasal symptoms in house dust mite (HDM) allergy, rhinorrhoea and nasal obstruction (0-3), were significantly reduced after updosing phase (V6-V1, mean + SD): Rhinitis score $-0.57+1.237$; Nasal obstruction $-0.78+1.126$; ${ }^{\star *} p \leq 0.005$ (b). For children, the improvement in the clinical parameters was even more pronounced (V6-V1, mean + SD): Rhinitis score: $-2.00+1.414$; Nasal obstruction: $(-1.00+1.000)$, however, due to the small number of patients these results could not reach significance (c). ns not significant

Germany, the sponsor of the study. C. Acikel declares that he has no competing interests.

Ethical standards The study upon which this analysis was based was approved by the Medical Ethics Committee of Vienna, Austria (reference number EK16-192-1016). The study was registered at clinical trials.gov with number NCT03127436. Informed consent was obtained from all individual participants included in the study.

Open Access This article is licensed under a Creative Commons Attribution 4.0 International License, which permits use, sharing, adaptation, distribution and reproduction in any medium or format, as long as you give appropriate credit to the original author(s) and the source, provide a link to the Creative Commons licence, and indicate if changes were made. The images or other third party material in this article are included in the article's Creative Commons licence, unless indicated otherwise in a credit line to the material. If material is not included in the article's Creative Commons licence and your intended use is not permitted by statutory regulation or exceeds the permitted use, you will need to obtain permission directly from the copyright holder. To view a copy of this licence, visit http://creativecommons.org/licenses/by/4.0/.

\section{References}

1. Roger A, Depreux N, Jurgens Y, Heath MD, Garcia G, Skinner MA. A novel and well tolerated mite allergoid subcutaneous immunotherapy: evidence of clinical and immunologic efficacy. Immun Inflamm Dis. 2014;2:92-8.

2. Roger A, Malet A, Moreno V, Parra A, Gutiérrez D, Lleonart R, et al. Real-life effect of a microcrystalline tyrosine adjuvanted mite immunotherapy in patients with allergic rhinitis. Immunotherapy. 2020;12:53-62.

3. Mosges R, Valero SA, Allekotte S, Jahed N, Astvatsatourov A, Sager A, et al. Subcutaneous immunotherapy with depig- 
mented-polymerized allergen extracts: a systematic review and meta-analysis. Clin Transl Allergy. 2019;9:29.

4. Shah-Hosseini K, Krudewig EM, Hadler M, Karagiannis E, Mosges R. Management of grass pollen allergy with 5-grass pollen tablet: results of a 2-year real-life study. Adv Ther. 2017;34:1382-97. 